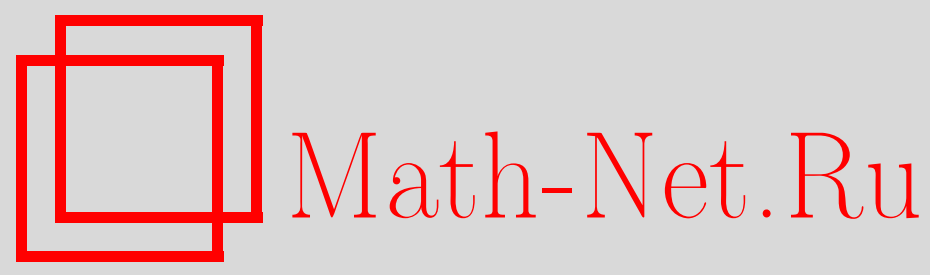

Ш. Т. Ишмухаметов, О вложении счетных порядков в тьюринговые степени, Матем. заметки, 2002, том 72, выпуск 5, 682-687

DOI: https://doi.org/10.4213/mzm456

Использование Общероссийского математического портала Math-Net.Ru подразумевает, что вы прочитали и согласны с пользовательским соглашением http://www.mathnet.ru/rus/agreement

Параметры загрузки:

IP : 18.208 .226 .222

26 апреля 2023 г., 15:13:14

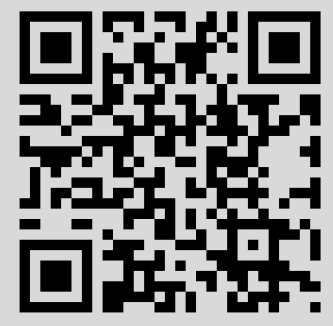




\title{
О ВЛОЖЕНИИ СЧЕТНЫХ ПОРЯДКОВ В ТЬЮРИНГОВЫЕ СТЕПЕНИ
}

\section{Ш. Т. Ишмухаметов}

\begin{abstract}
В классических работах Лермана, Лахлана и Лебеуфа разрабатьвается методвложения, позволяющий для различных частично упорядоченных структур строить изоморфные им начальные сегменты тьюринговых степеней. Мы проанализируем этот метод и докажем, что под каждой униформной в $\mathbf{0}^{\prime}$ убьвающей цепочкой степеней найдется ненулевая степень. Это накладывает ограничения на использование метода вложения.

Библиография: 10 названий.
\end{abstract}

Проблема строения начальных сегментов тьюринговых степеней $\mathbf{D}$ является одной из наиболее важных проблем в теории рекурсивных функций. Первоначальная конструкция минимальной степени Спектора [1] была упрощена и переложена на современный язык деревьев Шенфильдом [2]. Титгемайер [3] построил начальный сегмент $\mathbf{D}$, изоморфньй трехэлементной цепи, а Хьюджил [4] показал, что всякий счетньй линейный порядок изоморфен некоторому начальному сегменту степеней. В работах Лермана [5], [6] то же было доказано для конечных решеток. Из последнего результата следует, в частности, что теория тьюринговых степеней неразрешима. Наконец, в работе Лахлана и Лебеуфа [7] было доказано, что произвольная счетная верхняя полурешетка с наименьшим элементом изоморфна начальному сегменту D. Bсе достижения, полученные в этом направлении, были собраны вместе в монографии Лермана [8], где также описаны локальные (ниже $\mathbf{0}^{\prime}$ ) варианты этих теорем. В этой статье мы представляем новый результат, характеризующий метод вложения и накладьваюший определенные ограничения на его применение.

Определения всех используемых в статье понятий и обозначений можно найти в книге Cоара [9]. Через $\left\{\Phi_{i}, i \in \omega\right\}$ будем обозначать фиксированную геделевскую нумерацию всех частично рекурсивных функционалов. Множества мы будем отождествлять с их характеристическими функциями и для произвольного множества $A$ через $A \uparrow s$ обозначать начальный сегмент характеристической функции $A$ длины $s$.

Проанализируем метод вложения на примере произвольной бесконечной (счетной) структуры $L$. Требуется построить начальньй сегмент тьюринговых степеней, изоморфный $L$. Суть метода состоит в том, что непосредственно для каждого ненулевого элемента $a$ структуры $L$ строится методом деревьев множество $C_{a}$, тьюринговая степень которого и становится образом требуемого изоморфизма. Первьм шагом в решении

Работа выполнена при поддержке Российского фонда фундаментальных исследований, грант № 01-01-00733. 
этой задачи является получение подходящего представления $L$, под которым понимается произвольное отображение множества натуральных чисел $\omega$ на множество $|L|$.

ОПредЕЛЕниЕ 1 . Пусть $\mathbf{a}-$ степень. Структура $\langle L, \leqslant L\rangle$ назьвается а-npeдставимой, если существует представление $\nu$, при котором предикат

$$
R(i, j) \equiv \nu(i) \leqslant_{L} \nu(j)
$$

является рекурсивным в а. Степень $\mathbf{a}=\operatorname{deg}_{T} A$ называется сложсностью представления $\nu$. Если $\mathbf{a}=\mathbf{0}$, то представление называется рекурсивным.

Ясно, что если $\mathbf{a} \leqslant \mathbf{b}$ и структура $L$-представима, то $L$ также $\mathbf{b}$-представима. Задача состоит в том, чтобы найти представление $L$, имеюшее, по-возможности, меньшую сложность, желательно рекурсивную. Существуют, однако, естественные структуры, не имеющие рекурсивных представлений. Рассмотрим, для примера, структуру $L=\mathbf{D}$ $(\leqslant \mathbf{a})$, являющуюся главным идеалом полурешетки D. В общем виде эта структура $\mathbf{a}^{\prime \prime \prime}$-представима, и согласно результату Р. Шора для $\mathbf{a} \geqslant \mathbf{0}^{\prime}$ эта структура не может иметь представлений степени ниже $\mathbf{0}^{(4)}$.

Предположим теперь, что для рассматриваемой структуры $L$ уже найдено подходящее представление $\nu$. Рассмотрим другой аспект этой проблемы. Методом вложения определяется изоморфизм $\tau$ структуры $L$ на некоторый начальньй сегмент степеней $\mathbf{D}$. Фактически строится некоторая система множеств $S=\left\{C_{a}: a \in L\right\}$. Напомним, что для произвольных множеств $A$ и $B$ вьполняется $A \leqslant T$ ( $A$ сводится по Тьюрингу к $B)$ тогда и только тогда, когда найдется частично рекурсивньй функционал $\Phi$ такой, что $A=\Phi(B)$ (мы отождествляем множества $A$ и $B$ с их характеристическими функциями). Определим функцию сводимости $\operatorname{Red}(i, j)$ следующим образом: если $a=\nu(i)$ и $b=\nu(j)$ - элементы $L$ такие, что $a \leqslant L ~ b$, то $\operatorname{Red}(i, j))$ равно некоторому числу $m$ такому, что $C_{a}=\Phi_{m}\left(C_{b}\right)$, иначе $\operatorname{Red}(i, j) \uparrow$ (не определено). Неформально говоря, эта функция по паре $a, b, a \leqslant{ }_{L} b$, находит какой-нибудь алгоритм, сводящий множество $C_{a}$ к множеству $C_{b}$. В действительности же, подобная функция должна строится одновременно с системой множеств $S$, поскольку требование изоморфизма означает вьполнение условия

$$
a \leqslant L b \leftrightarrow C_{a} \leqslant{ }_{T} C_{b} .
$$

Спрашивается, какую сложность должна иметь функция Red? Кажется естественным предположение о том, что степень Red должна быть не вьше степени представления структуры $L$. Например, если $L$ - рекурсивно представимая структура, то кажется возможным подобрать рекурсивную систему сводящих алгоритмов. Ниже мы покажем, однако, что это не так и существуют рекурсивно представимые структуры, не имеющие вложений в тьюринговые степени с рекурсивной системой сводящих алгоритмов.

\section{1. Об убывающих цепочках степеней.}

ОПРЕДЕЛЕНИЕ 2. Пусть $\mathbf{a}-$ степень, а $P=\left\{\mathbf{c}_{0} \geqslant \mathbf{c}_{1} \geqslant \mathbf{c}_{2} \geqslant \cdots\right\}$ - невозрастающая последовательность тьюринговых степеней. Назовем последовательность $P$ униформной в $\mathbf{a}$, если существует функция $f$, рекурсивная в $\mathbf{a}$, такая, что для некоторого $C_{0} \in \mathbf{c}_{0}$ выполняется условие $(\forall n) C_{n+1}=\Phi_{f(n)}\left(C_{n}\right) \in \mathbf{c}_{n+1}$. Если функция $f$ рекурсивна, то назовем последовательность $P$ просто униформной.

В нашей основной теореме мы установим ограничение на вложение в $\mathbf{D}$ структур, содержащих бесконечные убывающие цепи. 
Теорема 1. $P=\left\{\mathbf{c}_{0} \geqslant \mathbf{c}_{1} \geqslant \mathbf{c}_{2} \geqslant \cdots>\mathbf{0}\right\}-$ униформная в $\mathbf{0}^{\prime}$ последовательность степеней. Найдется ненулевая степень с, находящаяся ниже каждой степени $\mathbf{c}_{n}, n \in \omega$.

Доказательству основной теоремы мы предпошлем несколько вспомогательных утверждений. Пусть $\{0\},\{1\},\{2\}, \ldots$ - геделевская нумерация всех частично рекурсивных функций. Строками будем назьвать элементы множества $\{0,1\}<\omega$, т.е. конечные цепочки, состоящие из нулей и единиц. Пусть $\langle e, x\rangle$ - номер пары $(e, x)$ в канторовской нумерации всех пар натуральных чисел. Определим нумерацию троек натуральных чисел $\langle e, x, y\rangle$, полагая $\langle e, x, y\rangle=\langle e,\langle x, y\rangle\rangle$. Определим множества $R_{e}$ для $e \in \omega$, полагая для $e \in \omega R_{e}=\{\langle e, x\rangle: x \in \omega\}$.

Лемма 1. Пусть е- произвольное число. Существует рекурсивный функиионал $\Phi$ такой, что для всех множеств $B$ функиия $\Phi(B)$ всюду определена, и если $B$ - нерекурсивное мнохсество, то $\Phi(B)$ конечно (как мнохсество) и найдется $x \in R_{e}$ такое, что $\Phi(B ; x) \neq\{e\}(x)$.

ДокАЗАТЕльство. Будем строить функционал $\Phi$ по шагам, как рекурсивно перечислимое множество пар строк $\langle\sigma, \tau\rangle$ такое, что

1) $\left\langle\sigma_{1}, \tau_{1}\right\rangle \in \Phi \wedge\left\langle\sigma_{2}, \tau_{2}\right\rangle \in \Phi \wedge \sigma_{1} \subset \sigma_{2} \rightarrow \tau_{1} \subset \tau_{2}$;

2) $\left\langle\sigma, \tau_{1}\right\rangle \in \Phi \wedge\left\langle\sigma, \tau_{2}\right\rangle \in \Phi \rightarrow \tau_{1}=\tau_{2}$.

Для этого на произвольном шаге $s>0$ для каждой строки $\sigma$ длины $|\sigma|=s$ мы определим конечное множество $W(\sigma)$, кодирующее некоторым образом начальные сегменты строки $\sigma$ и добавим к $\Phi$ пару $\langle\sigma, \rho\rangle$, где $\rho=W(\sigma) \uparrow(s+1)$.

Если множество $B$ нерекурсивно, то, кодируя в $\Phi(B)$ начальные сегменты $B$, мы тем самым увеличиваем сложность $\Phi(B)$, делаем его нерекурсивным, причем когда будет найден аргумент $x \in R_{e}$ такой, что $W(B)(x) \neq\{e\}(x)$, кодирование прекращается и множество $W(B)$ останется конечным.

Опишем инструкции произвольного шага $s+1$, предполагая, что для всех строк $\sigma$ длины $|\sigma| \leqslant s$ множества $W(\sigma)$ уже определены (для цепочки $\lambda$ нулевой длины множество $W(\lambda)$ по определению равно пустому множеству).

1. Пусть $\widetilde{\sigma}-$ произвольная цепочка длины $s+1$, а $\sigma$-ее начальньй сегмент длины $s$. Для произвольного $t \leqslant s$ определим функцию длины $l(\sigma, t)$ :

$$
l(\sigma, t)=\max \left\{z:\left(\forall x<z, x \in R_{e}\right) W\left(\sigma\lceil t)(x)=\{e\}_{t}(x)\right\} .\right.
$$

Назовем шаг $s+1(e, \sigma)$ - экспансиональным, если $l(\sigma, s)>\max _{t<s} l(\sigma, t)$.

2. Определим теперь $W(\widetilde{\sigma})$ равным $W(\sigma)$, если шаг $s+1$ не является $e$-экспансиональным, иначе определим $W(\widetilde{\sigma})$ равным объединению $W(\sigma)$ с числом $\langle e, s, k\rangle$, где $k-$ код строки $\sigma$ в некоторой фиксированной геделевской нумерации всех строк. Заметим, что в силу определения нумерации троек для всех $e, s$ и $k$ выполняется $\langle e, s, k\rangle>s$ и элемент, добавленный на шаге $s+1$ в $W(\sigma)$, превьшает $s$.

3. Добавим к функционалу $\Phi$ всевозможные пары $\langle\sigma, \rho\rangle$, где $|\sigma|=s+1$, а $\rho=W(\sigma) \uparrow$ $(s+1)$.

Описание конструкции окончено. Докажем некоторые ее свойства.

1. Множество $W(\sigma)$ определено для всех $\sigma$ и является подмножеством $R_{e}$.

Следует из конструкции и определения нумерации троек натуральных чисел. 
2. Функционал $\Phi$ определен корректно, т.е. для произвольных строк $\sigma_{1}$ и $\sigma_{2}$, если $\sigma_{1} \subset \sigma_{2}$ и $\left\langle\sigma_{1}, \tau_{1}\right\rangle \in \Phi,\left\langle\sigma_{2}, \tau_{2}\right\rangle \in \Phi$, то $\tau_{1} \subset \tau_{2}$.

Следует из того, что для строк $\sigma_{1} \subseteq \sigma_{2}$ вьполняется $W\left(\sigma_{1}\right) \subseteq W\left(\sigma_{2}\right)$, причем элементы, принадлежашие разности $W\left(\sigma_{2}\right)-W\left(\sigma_{1}\right)$ (если таковые найдутся), превьшают длину $\sigma_{1}$.

3. Пусть $B$ - нерекурсивное множество. Тогда множество $\Phi(B)$ конечно и $\left(\exists x \in R_{e}\right)$ $\Phi(B ; x) \neq\{e\}(x)$.

Предположим, что утверждение неверно и для всех $x \in R_{e} \Phi(B ; x)=\{e\}(x)$. В силу того, что $\Phi(B)$ является подмножеством $R_{e}$, множество $\Phi(B)$ является рекурсивным. Для произвольного $s$ обозначим через $\sigma_{s}$ начальньй интервал $\Phi(B)$ длины $s$. Ясно, что в силу предположения множество $\operatorname{Exp}(e)=\left\{s:\right.$ шаг $s+1\left(e, \sigma_{s}\right)$-экспансиональньй $\}$ является бесконечньм и рекурсивным. Тогда бесконечно и множество $\Phi(B)$, поскольку на каждом $\left(e, \sigma_{s}\right)$-шаге $s+1$ в $W\left(\sigma_{s}\right)$ добавляется новьй элемент $z \neq 0$, которьй на шаге $z+1$ попадет в множество $\Phi(B)$. Но, зная элементы множества $\Phi(B)$, мы можем восстановить начальные сегменты $B$ произвольной длины, и $B$ окажется рекурсивным в противоречие с условием леммы.

Этим завершается доказательство леммы.

Заметим, что поскольку инструкции для функционала $\Phi$ эффективно зависят от $e$, то справедливо более сильное утверждение.

Лемма 2. Существует общерекурсивная функиия $h$ такая, что для всех множеств $A$ и $е \in \omega$ функиионал $\Phi_{h(e)}(A)$ всюду определен и содержится как множсество в $R_{e}$, причем если $A$ нерекурсивно, то $\Phi_{h(e)}(A)$ конечен (как множсество) $u\left(\exists x \in R_{e}\right) \quad \Phi_{h(e)}(A ; x) \neq\{e\}(x)$.

ПРЕДЛОЖЕНИЕ 1. Пусть $P=\left\{\mathbf{c}_{0} \geqslant \mathbf{c}_{1} \geqslant \mathbf{c}_{2} \geqslant \cdots\right\}-$ униформная последовательность степеней. Найдется ненулевая степень с, находящаяся ниже каждой степени $\mathbf{c}_{n}, n \in \omega$.

ДокАЗАТЕЛЬСТВо. Пусть функция $f$ и множества $C_{n}, n \in \omega$, такие же, как в определении униформной последовательности, а функция $h$ взята из последней леммы. Определим множество $C=\bigcup_{n \in \omega} \Phi_{h(n)}\left(C_{n}\right)$. Очевидно, $C$ нерекурсивно. Покажем, что $C \leqslant_{T} C_{n}$ для любого $n$. Действительно, для любого $e \in \omega$ множество $\Phi_{h(e)}\left(C_{e}\right)$ конечно. Пусть $\langle e, z\rangle-$ произвольное число такое, что $e>n$. Чтобы узнать $\langle e, z\rangle \in C$, надо узнать $\langle e, z\rangle \in C_{e}$, а поскольку $C_{e}$ униформно рекурсивно в $C_{n}$, то последний вопрос можно переадресовать оракулу $C_{n}$. Предложение доказано.

ПРЕДЛОЖЕНИЕ 2. Пусть $P=\left\{\mathbf{c}_{0} \geqslant \mathbf{c}_{1} \geqslant \mathbf{c}_{2} \geqslant \cdots\right\}-$ последовательность степеней. Если $P$ униформна в $\mathbf{0}^{\prime}$, то $P$ просто униформна.

ДокАЗАТЕЛЬСтво. Пусть функция $f \leqslant \mathbf{0}^{\prime}$ такая же, как в определении 2 , т.е. найдется цепочка множеств $\left\{C_{n}\right\}, n \in \omega$, такая, что для всех $n C_{n} \in \mathbf{c}_{n}$ и $C_{n+1}=\Phi_{f(n)}\left(C_{n}\right)$. Сушествует двухместная общерекурсивная функция $f(s, x)$ такая, что для всех $n f(n)=$ $\lim _{s} f(s, n)$. Пусть $m(n)=\mu t[t \geqslant m(n-1) \wedge(\forall s \geqslant t) f(s, n)=f(n)]$ - модуль функции $f(s, n)$ (по определению $m(-1)$ равно 0).

По последовательности $\left\{C_{n}\right\}, n \in \omega$, и функции $f$ мы будем строить униформную последовательность множеств $\left\{\widetilde{C}_{n}\right\}, n \in \omega$, такую, что каждое $\widetilde{C}_{n} \in \mathbf{c}_{n}$. Определим $\widetilde{C}_{0}=C_{0}$. 
Предполагая оракул $C_{0}$ заданньм, определим для произвольных $s$ и $x$ аппроксимацию $C_{1}^{s}(x)$ значения $C_{1}(x)$. Для этого найдем наименьшее число $t \geqslant \max \{s, x\}$ такое, что $\Phi_{f(t, 0), t}\left(C_{0} ; x\right) \downarrow$ и определим $C_{1}^{s}(x)=\Phi_{f(t, 0), t}\left(C_{0} ; x\right)$.

В силу определения для любых $x$ и $s \geqslant m(0)$ значение $C_{1}^{s}(x)$ совпадает со значением $C_{1}(x)$.

Определим множество $\widetilde{C}_{1}$, полагая $\widetilde{C}_{1}(\langle x, s\rangle)$ равным по определению $C_{1}^{s}(x)$.

Очевидно, что для всех $x \geqslant m(0)$ столбец $\widetilde{C}_{1}^{(x)}$ целиком состоит из значений $C_{1}(x)$, а столбцы $\widetilde{C}_{1}^{(x)}$ для $x<s_{0}$ могут содержать лишь конечное число элементов, отличных от $C_{1}(x)$. Поэтому множества $C_{1}$ и $\widetilde{C}_{1} T$-эквивалентны.

Пусть теперь $n \geqslant 1$ - произвольное число. Предположим, что множество $\widetilde{C}_{n}$ уже построено, причем для всех $s \geqslant m(n)$ и произвольных $x \widetilde{C}_{n}(x, s)=C_{n}(x)$, а для $x \geqslant m(n)$ столбцы $\widetilde{C}_{n}^{(x)}$ полностью состоят из одного значения $C_{n}(x)$.

1. Для каждого $s \in \omega$ определим функцию $C_{n}^{s}$, полагая ее значение $C_{n}^{s}(x)$ в произвольной точке $x$ равным $\widetilde{C}_{n}(x, s)$. В силу индукционного предположения для $s \geqslant m(n)$ функции $C_{n}^{s}$ совпадают с характеристической функцией множества $C_{n}$.

2. Определим множество $\widetilde{C}_{n+1}$, вычисляя значение $\widetilde{C}_{n+1}(\langle x, s\rangle)$ следующим образом: находим наименьшее $t \geqslant \max \{s, x\}$ такое, что $\Phi_{f(s, n), t}\left(C_{n}^{t} ; x\right) \downarrow$, и полагаем

$$
\widetilde{C}_{n+1}(x, s)=\Phi_{f(s, n), t}\left(C_{n}^{t} ; x\right)
$$

Нетрудно доказать, что множество $\widetilde{C}_{n+1}$ удовлетворяет индукционному предположению, сформулированному вьше, поэтому можно продолжить процедуру определения последовательности $\left\{\widetilde{C}_{n}\right\}$ индуктивно. Пусть последовательность $\left\{\widetilde{C}_{n}\right\}$ уже построена. В силу своей структуры каждое $\widetilde{C}_{n} T$-эквивалентно $C_{n}$. Кроме того, алгоритм, сводящий $\widetilde{C}_{n+1}$ к $\widetilde{C}_{n}$, определяется эффективно по номеру $n$.

Этим завершается доказательство предложения.

Доказательство теоремы следует непосредственно из предложений 1 и 2.

2. Выводы. Пусть $L$ обозначает структуру $\omega^{*}$, т.е. линейньй порядок, обратньй натуральному ряду. Согласно теореме Хьюджилла [4] $L$ изоморфна некоторому начальному сегменту степеней $\mathbf{D}\left(\leqslant \mathbf{0}^{\prime \prime}\right)$, а по теореме Лермана [8, XII.5.1] изоморфна некоторому сегменту степеней ниже $\mathbf{0}^{\prime}$. Пусть $P=\left\{\mathbf{c}_{0}>\mathbf{c}_{1}>\mathbf{c}_{2}>\cdots>\mathbf{0}\right\}-$ цепь степеней, являющаяся образом $L$ в $\mathbf{D}$. Согласно нашей теореме эта последовательность не может быть униформной в $\mathbf{0}^{\prime}$, иначе найдется ненулевая степень с, находящаяся ниже всех $\mathbf{c}_{n}, n \in \omega$.

Анализ доказательства Хьюджилла показьвает, что конструируемая им последовательность $P$ является униформной в $\mathbf{0}^{\prime \prime}$. Действительно, на каждом шаге $n$ конструкции Хьюджилла рассматривается конечньй линейньй подпорядок $L_{n}$ структуры $L$ и методом тотальных униформных деревьев (см. Одифредди [10]) строится система деревьев $T_{k}, k<n$, являющихся поддеревьями деревьев, построенньх на предыдушем шаге. Особенность конструкции такова, что построение деревьев производится 0" но. На следующем шаге конструкции новые элементы подпорядка $L_{n+1}$, отсутствующие в $L_{n}$, кодируются уже в эти вновь определенные деревья. Значит, индексы сводящих алгоритмов, определяемых на шаге $n+1$, зависят от индексов деревьев $T_{k}, k<n$, которые и определяются $\mathbf{0}^{\prime \prime}$-рекурсивно. 
Таким образом, наша теорема дает наилучший результат, характеризующий убывающие цепочки степеней, и является неверной, например, для порядков, униформно рекурсивных в $\mathbf{0}^{\prime \prime}$. В свою очередь, метод вложения также не может строить последовательности сводящих алгоритмов рекурсивно или с использованием оракула $\mathbf{0}^{\prime}$.

Заметим, что в монографии Одифредди [10, с. 527-528]приводится упрощенная схема доказательства теоремы Хьюджилла, дающая униформную последовательность сводящих алгоритмов и потому являющаяся неверной и противоречащей нашей теореме.

Автор благодарен М. М. Арсланову и И.Ш. Калимуллину за ряд ценных замечаний, способствовавших улучшению доказательств.

\section{СПИСОК ЦИТИРОВАННОЙ ЛИТЕРАТУРЫ}

[1] Spector C. On degrees of recursive unsolvability // Ann. of Math. 1956. V. 64. P. 581-592.

[2] Shoenfield J. R. A theorem on minimal degrees // J. Symbolic Logic. 1966. V. 31. P. 539-544.

[3] Titgemeyer D. Untersuchungen über die Struktur des Kleene-Postchen Halbverbandes des Grade der Rekursivenunlösbarkeit // Arch. Math. Logik Grundlagenforsch. 1962. V. 8. № 1-2. P. 45-62.

[4] Hugill D.F. Initial segments of Turing degrees // Proc. London Math. Soc. 1969. V. 19. P. 1-16.

[5] Lerman M. Some nondistributive lattices as initial segments of the degrees of unsolvability // J. Symbolic Logic. 1969. V. 34. P. 85-98.

[6] Lerman M. Initial segments of the degrees of unsolvability // Ann. Math. 1971. V. 93. P. 365-389.

[7] Lachlan A. H., Lebeuf R. Countable initial segments of the degrees of unsolvability // J. Symbolic Logic. 1976. V. 41. P. 289-300.

[8] Lerman M. Degrees of Unsolvability, Perspectives in Mathematical Logic. Omega Series. Berlin-Heidelberg-London-New York-Tokyo: Springer-Verlag, 1983.

[9] Соар Р. И. Вычислимо перечислимые множества и степени. Казань, 2000.

[10] Odifreddi P. Classical Recursion Theory. Studies in Logic and the Foundations of Mathematics. V. 125. Amsterdam-New York-Oxford-Tokyo: North-Holland, 1989. 